\title{
Endotoxaemia as a cause of fever in immunosuppressed patients
}

\author{
RI HARRIS, PCW STONE, GR EVANS, J STUART \\ From the Department of Haematology, The Medical School, University of Birmingham, Birmingham B15 \\ $2 T J$
}

SUMMARY Using a recently developed chromogenic substrate assay sensitive to $10 \mathrm{pg} / \mathrm{ml}$ Escherichia coli endotoxin in plasma, systemic endotoxaemia was found in $52 \%$ of 21 episodes of fever in patients with a haematological malignancy who were infected. Endotoxaemia was also found in $27 \%$ of 22 episodes of fever of unknown origin. In 45 afebrile patients neither neutropenia nor cytotoxic chemotherapy was a cause of endotoxaemia. Passage of endotoxin from portal blood into the systemic circulation can contribute to unexplained fever in immunosuppressed patients.

In man the normal bowel flora constitutes a large reservoir of bacterial endotoxin with the potential to cause portal venous endotoxaemia.' Any endotoxin present in portal blood is normally destroyed by peroxidases and esterases present in hepatic fixed macrophage (Kupffer) cells and thus does not reach the systemic circulation. ${ }^{2}$ In cirrhosis and in Reye's syndrome, however, systemic endotoxaemia without bacteraemia has been shown ${ }^{13}$ and has been ascribed to Kupffer cell dysfunction ${ }^{4}$ or intrahepatic portal-systemic shunting of blood. ${ }^{\circ}$

In haematological malignancy systemic endotoxaemia without bacteraemia could occur as a consequence of intestinal mucosal damage by cytotoxic agents, or because of a numerical or functional defect of neutrophils, or because of impaired hepatic reticuloendothelial cell clearance of endotoxin. Episodes of fever are common in these patients and often remain unexplained despite intensive microbiological investigation. We have therefore determined the incidence of endotoxaemia, as measured by a new chromogenic substrate assay, in patients with haematological malignancy and have studied the relation between endotoxaemia and the development of fever.

\section{Patients and methods}

\section{PATIENTS}

Endotoxin assays were carried out on 27 healthy subjects (18 men, nine women) and 32 outpatients

Accepted for publication 2 December 1983
(10 men, 22 women) with a non-malignant haematological disorder. One hundred patients ( 54 men, 46 women) with the following haematological malignancies were also studied: acute myeloblastic leukaemia (21 patients), acute lymphoblastic leukaemia (13 patients), multiple myeloma (20 patients), chronic lymphocytic leukaemia (26 patients), and lymphoma or macroglobulinaemia (20 patients). Sixty of these were outpatients who were not receiving chemotherapy and were not neutropenic. Several patients were studied on more than one occasion as their clinical circumstances changed.

\section{METHODS}

After cleaning the skin with $70 \%$ isopropanol, heparinised blood $(25 \mathrm{IU} / \mathrm{ml}$; Weddel Pharmaceuticals Ltd, London) was collected into sterile plastic universals (Sterilin Ltd, Teddington) for endotoxin assay. All heparin batches were checked for endotoxin contamination before use. During episodes of fever additional blood samples were taken for $\mathrm{C}$ reactive protein estimation, viral antibody titres, and blood cultures (aerobic and anaerobic). Urine and, where possible, sputum samples were also obtained for culture. Febrile patients underwent daily clinical examination and additional investigations (for example, bronchoscopy) were performed when clinically indicated.

Plasma endotoxin assays were carried out as described previously ${ }^{7}$ using a class II microbiological safety cabinet and new, endotoxin free tubes for each determination. Unseparated blood specimens 
were kept at $4^{\circ} \mathrm{C}$ and processed within $2 \mathrm{~h}$ of venepuncture. Briefly, platelet poor plasma was diluted $1 / 10$ in sterile, pyrogen free water and heated at $75^{\circ} \mathrm{C}$ for $5 \mathrm{~min}$ to remove plasma inhibitors. The diluted, heated sample was incubated with Limulus amoebocyte lysate ("Pyrogent" Mallincrodt, St Louis, Missouri) for $45 \mathrm{~min}$ at $37^{\circ} \mathrm{C}$ before adding chromogenic substrate S2423 (Kabi Diagnostica, Stockholm). The chromogenic reaction was stopped after exactly $3 \mathrm{~min}$ and read spectrophotometrically at $405 \mathrm{~nm}$. Results were reported as the optical density (OD) reading obtained after the subtraction of both test blank and reagent blank readings. ${ }^{7} \mathrm{C}$ reactive protein estimations were performed by a laser nephelometric method, ${ }^{8}$ total leucocyte counts on EDTA blood were obtained using a Coulter Model S (Coulter Electronics Ltd, Luton), and differential counts were performed manually. Statistical analysis was carried out using the Mann-Whitney $\mathrm{U}$ test.

\section{Results}

The 27 healthy subjects all gave endotoxin assay values in the range $0-0 \cdot 15$ OD units. The same reference range was obtained for 31 of the 32 outpatients with a non-malignant haematological disorder and 58 of the 60 outpatients with a haematological malignancy who were not taking cytotoxic drugs and were not neutropenic. These three exceptions (endotoxin values in the range $0 \cdot 17-0 \cdot 25$ OD units) are shown in Fig. 1, where the three groups have been amalgamated to give a reference group of 119 individuals. To determine the effects of neutropenia and cytotoxic chemotherapy on this reference range endotoxin assays were performed on 75 plasma specimens from 45 afebrile outpatients with a haematological malignancy, who were subdivided as follows:

Group I-currently receiving cytotoxic agents but not severely neutropenic (mean neutrophil count $3.7 \times 10^{9} / 1$; range $1 \cdot 1-6 \cdot 8$ )

Group II-neutropenic (mean neutrophil count $0 \cdot 4$ $\times 10^{4} / \mathrm{l}$; range $0-0 \cdot 9$ ) but no cytotoxic agents given for at least three days before study

Group III-currently receiving cytotoxic agents and also neutropenic (mean neutrophil count $0.4 \times 10^{\%} /$ $\mathrm{l}$; range $0-0 \cdot 9$ )

There was no significant difference $(p>0 \cdot 1)$ in plasma endotoxin values between these three groups or between any one of them and the reference group (Fig. 1). Only two results above 0.15 OD units were obtained $(0 \cdot 16$ and 0.17 OD units in group I). Thus the reference range of $0-0 \cdot 15$ OD units for healthy subjects was also applicable to $98 \%$ of the 194 test results obtained for all the afebrile patients in Fig. 1.

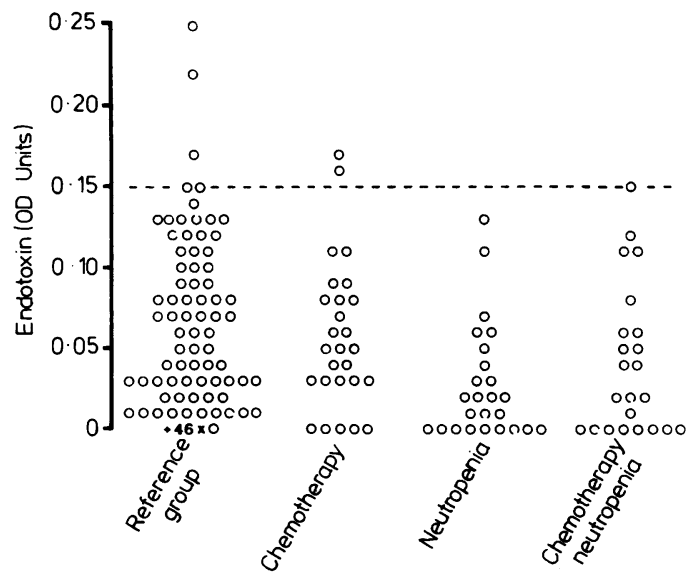

Fig. 1 Endotoxin assay results (OD units) for reference group (119 individuals) compared with 27 specimens from non-neutropenic patients currently receiving cytotoxic agents (group I), 25 specimens from neutropenic patients not currently receiving chemotherapy (group II), and 23 specimens from patients currently receiving chemotherapy who were also neutropenic (group III). Interrupted line shows upper limit of reference range incorporating $98 \%$ of all 194 test results and $100 \%$ of results for the 27 healthy individuals.

Endotoxin assays were performed within $24 \mathrm{~h}$ of the onset of 43 episodes of fever (mean oral temperature $38.4^{\circ} \mathrm{C}$, range $37.5-39.8^{\circ} \mathrm{C}$ ) in 29 inpatients with haematological malignancy. In 21 episodes of fever in which there was clinical or laboratory evidence of infection, or both, 11 showed plasma endotoxin activity greater than $0 \cdot 15$ OD units (Fig. 2 ). These episodes comprised Gram negative septicaemia ( 5 cases), Gram positive septicaemia (2 cases), respiratory infection (2 cases), and unidentified infection causing a rise in $C$ reactive protein concentration to greater than $150 \mathrm{mg} / \mathrm{l}(2$ cases). An episode of unexplained fever was defined as: absence of clinical signs of infection together with negative bacteriological cultures, maximum serum $C$ reactive protein concentration less than $100 \mathrm{mg} / \mathrm{l}$, negative viral antibody titres, and no temporal relation to administration of blood products. Twenty two such episodes were identified and six had endotoxin assay results greater than 0.15 OD units (Fig. 2).

Serial endotoxin assays were undertaken during 12 episodes of fever showing initial endotoxin values of more than 0.15 OD units; endotoxaemia and fever failed to resolve in parallel in 11 of these episodes (Fig. 3). In the six endotoxaemic patients with unexplained fever, endotoxin assay results fell to less than $0.15 \mathrm{OD}$ units within $48 \mathrm{~h}$; in three of these patients the fever also resolved within $48 \mathrm{~h}$ but 


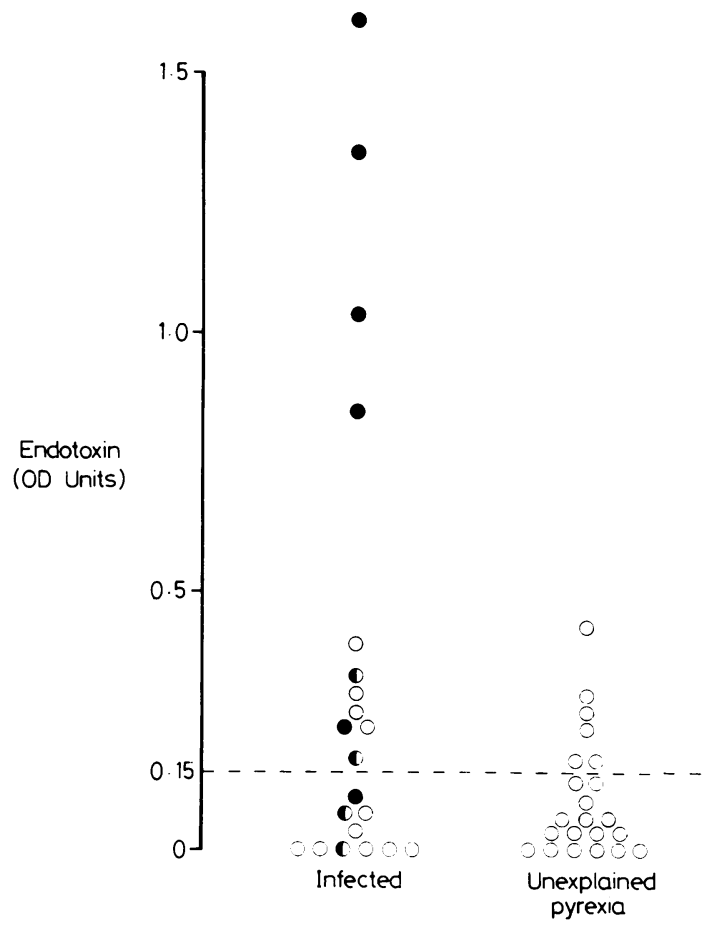

Fig. 2 Endotoxin assay results (OD units) in 43 episodes of fever: 21 with evidence of infection (1-Gram negative septicaemia; -Gram positive septicaemia; $\bigcirc-$ other infection) and 22 unexplained. Interrupted line shows upper limit of endotoxin reference range as in Fig. 1.

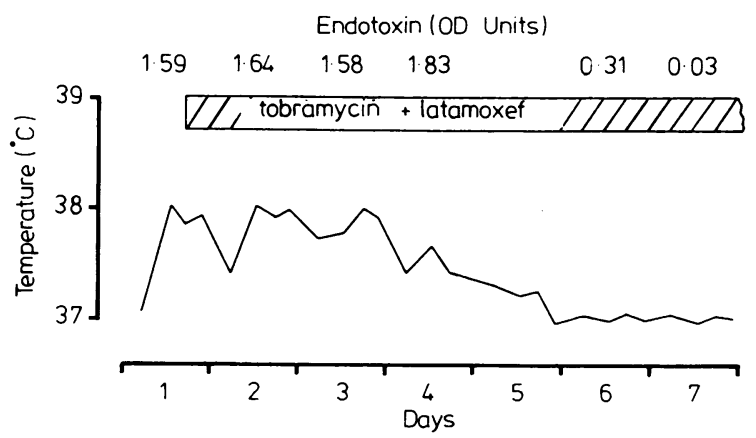

Fig. 3 Persistence of endotoxaemia after resolution of fever in a patient from whom Pseudomonas aeruginosa was isolated from day 1 blood cultures.

in two it was sustained for five to seven days. Study of seven episodes of clinically evident infection without fever showed transiently raised endotoxin activity in five. A close temporal relation between endotoxaemia and fever was not therefore established in any of these groups.

\section{Discussion}

Most of the earlier clinical studies of endotoxaemia used the Limulus gelation assay, which has the disadvantages of poor specificity, subjective end point, and relative lack of sensitivity. The improved chromogenic substrate assay developed for the present study links the activation of the Limulus amoebocyte proenzyme to a chromogenic substrate, thus bypassing the gelation reaction. This assay is more sensitive (to $10 \mathrm{pg} / \mathrm{ml}$ Escherichia coli endotoxin in plasma), specific, and reproducible (within batch coefficient of variation of $4 \%$ ). ${ }^{7}$ Contamination with extraneous endotoxin, which may have occurred in earlier studies, was minimised by performing the assay in a laminar air flow cabinet and by using new, endotoxin free tubes for each test.

Our studies in 45 afebrile patients suffering from haematological malignancy suggest that normal mechanisms for the prevention of systemic endotoxaemia usually remain intact despite neutropenia or cytotoxic chemotherapy. In febrile patients, however, an increase in endotoxin activity (range $0.17-$ 1.59 OD units) was seen in 11 of $21(52 \%)$ episodes of fever in infected patients and a smaller increase (range $0 \cdot 17-0.43$ OD units) in six of $22(27 \%$ ) episodes in patients with unexplained fever. The latter result is similar to that of a recent Dutch study, in which endotoxaemia was detected in $25 \%$ of 55 blood samples obtained during unexplained fever in children with acute lymphoblastic leukaemia." The less sensitive Limulus amoebocyte lysate gelation assay was used in the Dutch study, however, and it is likely that some of our patients would have shown negative results by this method.

Systemic endotoxaemia was found in two of our patients who had Gram positive bacteraemia. It has been suggested ${ }^{10}$ that non-specific activation of the Limulus proenzyme can occur in the presence of peptidoglycan derived from Gram positive organisms, but this has been disputed." An alternative explanation is functional reticuloendothelial blockade induced by the bacteraemia, causing endotoxaemia as a secondary event.

Serial study of patients with endotoxaemia showed an inconstant temporal relation with fever. Possible reasons for this include the variation in pyrogenicity between different endotoxins, ${ }^{2}$ development of host tolerance to endotoxin," and the presence of other pyrogens. Patients with unexplained fever and endotoxaemia may have had an undiagnosed Gram negative bacterial infection. Alternatively, some other occult infection could have caused Kupffer cell dysfunction, by antigen blockade, with endotoxaemia occurring as a secondary event. Damage to Kupffer cells by cytotoxic 
drugs may also cause secondary endotoxaemia in the absence of infection.

This study of a chromogenic substrate assay for endotoxin has established a reference range of $0-$ (). 15 OD units which was representative of $98 \%$ of 194 assays performed on both healthy subjects and afebrile patients with and without a haematological malignancy. The raised plasma endotoxin concentrations found in $52 \%$ of febrile infected patients and in $27 \%$ of episodes of unexplained fever indicate that endotoxaemia is a contributory factor to fever in immunosuppressed patients.

We are indebted to the Leukaemia Research Fund for financial support and to the West Midlands Rotational Training Programme in Haematology for sabbatical leave for one of us (RIH).

\section{References}

' Prytz H, Holst-Christensen J, Korner B. Liehr H. Portal venous and systemic endotoxaemia in patients without liver disease and systemic endotoxaemia in patients with cirrhosis. Scand $J$ Gastroenterol 1976;11:857-63.

2 Filkins JP. Hepatic lysosomes and the inactivation of endotoxin. J Reticuloendothel Soc 1971;9:480-90.

${ }^{3}$ Cooperstock MS, Tucker RP, Baublis JV. Possible pathogenic role of endotoxin in Reye's syndrome. Lancet 1975:i: 1272-4.

+ Bradfield JWB. Control of spillover. The importance of Kupffer cell function in clinical medicine. Lancet 1974;ii:883-6.
Gonzalez-Calvin J, Scully MF, Sanger Y, et al. Fibronectin in fulminant hepatic failure. $\mathrm{Br}$ Med J 1982:285:1231-2.

- Wolter J, Liehr H. Grün M. Hepatic clearance of endotoxins: differences in arterial and portal venous infusion. Reticuloendothel Soc 1978:23:145-52.

' Harris RI, Stone PCW, Stuart J. An improved chromogenic substrate endotoxin assay for clinical suse. J Clin Pathol 1983:36: 1145-9.

${ }^{\star}$ Rose PE. Johnson SA, Meakin M. Mackie PH. Stuart J. Serial study of $C$-reactive protein during infection in leukaemia. $J$ Clin Pathol 1981:34:263-6.

${ }^{4}$ Dankert J, de Vries JA. Veninga TS. Intestinal endotoxin and the occurrence of unexplained fever in leukaemic patients during treatment with cyclophosphamide. In: Fliedner TM. Heit $\mathrm{H}$, Niethammer D, Pflieger $\mathrm{H}$, eds. Clinical and experimental gnotobiotics, Zbl Bakt Suppl 7. New York: Gustav Fischer Verlag. 1979:369-76.

"' Wildfeuer A, Heymer B, Schleifer KH, Haferkamp O. Investigations on the specificity of the Limulus test for the detection of endotoxin. Appl Microbiol 1974;28:867-71.

"Levin J. The Limulus test and bacterial endotoxins: some perspectives. In: Watson SW. Levin J, eds. Endotoxins and their detection with the Limulus amebocyte lysate test. New York: Alan R Liss, 1982;7-24.

12 Heishman J, Fowlkes JA. A comparison of pyrogenicity of bacterial endotoxins from a variety of Gram-negative bacteria as determined by the LAL test. In: Watson SW, Levin J, eds. Endotoxins and their detection with the Limulus amebocyte lysate test. New York: Alan R Liss, 1982;131-40.

Requests for reprints to: Professor J Stuart, Department of Haematology, The Medical School, University of Birmingham, B15 2TJ, England. 